\title{
Multilinguales
}

\section{Origines des interférences interlinguales lexicales dans les productions écrites des apprenants de FLE}

Origins of interlingual lexical interferences in the written expressions of learners

of French as a foreign language

\section{Assia Laidoudi}

\section{(2) OpenEdition}

\section{Journals}

Édition électronique

URL : https://journals.openedition.org/multilinguales/4723

DOI : 10.4000 /multilinguales.4723

ISSN : 2335-1853

Éditeur

Université Abderrahmane Mira - Bejaia

Référence électronique

Assia Laidoudi, « Origines des interférences interlinguales lexicales dans les productions écrites des apprenants de FLE », Multilinguales [En ligne], 13 | 2020, mis en ligne le 15 juillet 2020, consulté le 18 mai 2021. URL : http://journals.openedition.org/multilinguales/4723 ; DOI : https://doi.org/10.4000/ multilinguales. 4723

Ce document a été généré automatiquement le 18 mai 2021.

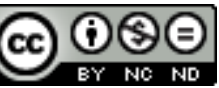

Multilinguales est mise à disposition selon les termes de la Licence Creative Commons Attribution Pas d'Utilisation Commerciale - Pas de Modification 4.0 International 


\section{Origines des interférences interlinguales lexicales dans les productions écrites des apprenants de FLE}

Origins of interlingual lexical interferences in the written expressions of learners

of French as a foreign language

Assia Laidoudi

1 Dans leur chemin vers l'apprentissage des langues étrangères, les apprenants croisent des difficultés qui se manifestent sous forme d'erreurs interlinguales ou intralinguales. Öztokat (1993) se penche sur la distinction entre ces deux catégories d'erreurs. Selon lui, les premières sont dues au contact des langues en présence alors que les secondes relèvent de la langue cible elle-même. Les erreurs interlinguales, auxquelles nous nous intéresserons dans la présente étude, sont dues essentiellement à un transfert négatif, engendrant ce que nous désignons par interférences. Pourtant inhérentes à tout processus d'acquisition, étant des traces des interlangues des apprenants (Robert, 2002 ; Cuq \& Gruca, 2005...), le regard que leur porte certains auteurs ne passe pas inaperçu dans la mesure où ils ne les admettent pas aisément. Dans ce contexte, Medane (2015) et Hamers (1997) appréhendent ces interférences comme des indicateurs d'une incompétence linguistique. Quel serait le regard le plus judicieux à porter sur ces erreurs?

2 Cette première question, à laquelle nous tenterons de répondre, ne constitue pas la problématique de notre étude centrée sur les origines des interférences interlinguales dans les productions écrites en FLE. De tous les types d'interférences, nous analyserons les interférences lexicales, plus fréquentes selon Klinkenberg (1999); ce qui permettra de dévoiler si ces interférences sont courantes dans les écrits des apprenants du cycle moyen, population de notre étude. Ces apprenants, vu la complexité de la situation linguistique de notre pays, sont confrontés à trois langues introduites à des moments distincts de leur parcours scolaire. La présence de ces trois langues dans leur 
environnement linguistique pourrait générer des interférences lexicales dont les origines sont à comprendre pour une meilleure intervention pédagogique. La littérature scientifique a tendance à attribuer ces interférences lexicales à la première langue des apprenants qui conditionne leurs futurs apprentissages (Medane, 2015; Galisson \& Coste, 1976). Les erreurs de nos apprenants seraient, dans ce cas-ci, engendrées par la langue arabe, leur langue maternelle (LM). Mais, cette langue seraitelle la seule source des interférences interlinguales lexicales dans leurs productions écrites en FLE? Cette question s'impose si nous ne négligeons pas la présence de l'anglais, la deuxième langue étrangère (LE2) apprise ultérieurement, qui présente des similitudes lexicales -historiquement justifiées- avec le français; ce qui pourrait créer des confusions et des erreurs.

3 Une telle situation nous mène à supposer que les erreurs commises par les apprenants dans leurs productions ne se réduisent plus à des transferts négatifs de la langue maternelle et que la langue étrangère provoque des interférences lexicales.

4 En vue de vérifier ces hypothèses, nous étudierons les productions écrites des apprenants du cycle moyen. Nous procéderons à une analyse des interférences interlinguales lexicales pour en déterminer l'origine : l'arabe (la LM) ou l'anglais (LE2). Ces données, nous les investirons pour percer le mystère de ces erreurs étant donné que les comprendre contribue à mieux enseigner.

\section{Pour comprendre les interférences interlinguales lexicales}

5 Cités par Agues Martins, Besse et Porquier abordent l'importance d'étudier les interférences dont la compréhension contribue à

mieux comprendre les processus d'apprentissage d'une langue étrangère (1991, In

Agues Martins, $2007: 40$ ).

Une telle étude assure une amélioration certaine des pratiques enseignantes et une installation de compétences réelles, et ce, en exploitant les erreurs en tant qu'outils d'apprentissage. A quelles questions faut-il répondre pour comprendre ces interférences?

\section{Qu'est-ce qu'une interférence?}

6 Le concept «interférence » est défini par de nombreux auteurs. Kannas (1994) et Klinkenberg (1999) désignent par interférence tout emploi des traits (phonétiques, lexicaux, syntaxiques...) de la première langue dans une langue cible. Etant donné que cet emploi transgresse les normes de la langue cible, Hassan (1974) et Hamers (1997) conçoivent cette interférence comme une transgression, certes involontaire, des règles de la langue cible, autrement dit, une « violation inconsciente d'une norme d'une langue par l'influence des éléments d'une autre langue » (Hassan, 1974 :171).

A ces conceptions -certainement utiles- nous préférons les définitions de l'interférence qui privilégient le point de vue didactique. Ainsi, Hamers et Blanc (1983) définissent ces interférences comme

des problèmes d'apprentissage dans lesquels l'apprenant transfère le plus souvent inconsciemment et de façon inappropriée des éléments et des traits d'une langue connue dans la langue cible (In Agues Martins, 2007 : 41). 
7 La difficulté des apprenants réside ainsi dans leur incapacité à transférer adéquatement leurs connaissances dans la première langue pour résoudre les situations-problèmes de la langue cible. Ceux-ci recourent, inconsciemment, et parfois consciemment (Coste ; Galisson, 1988), aux éléments connus pour compenser les éléments inconnus, ce qui conduit souvent à des incorrections vu les différences qui existent entre les deux systèmes linguistiques en présence. Soulignons que la langue source pourrait dans certains cas être influencée par les langues apprises ultérieurement. Dans cet ordre d'idées, Cuq (2003) considère que les interférences ne se manifestent pas seulement en langue étrangère dans la mesure où la langue maternelle des apprenants pourrait être empreinte des traits des langues étrangères apprises. Pour illustrer ses propos, Cuq cite l'exemple du mot " pastique » (mot français) qui pourrait être transcrit «plastic » (mot anglais) par les apprenants, par contamination.

En plus de la définition de Hamers et Blanc (1983), nous citons celle de Weinrich (1953) qui estime que ce phénomène -relatif à un changement au niveau des structures d'une langue- est dû à une intrusion de traits inappropriés; d'où les interférences. Leur nombre dans les discours produits est déterminé par le degré de différence entre les langues: plus les différences sont nombreuses, plus les interférences le sont. La définition de cet auteur, qui met également la lumière sur la conception didactique du concept, insiste sur la raison essentielle des interférences constatées: un transfert négatif dû au contact des langues notamment différentes.

Evoquer le concept de transfert négatif nous mène à aborder celui de transfert positif. Que les apprenants s'appuient sur ce qui est connu pour assimiler ou maîtriser ce qui est inconnu est parfois utile. Nos propos seront justifiés si nous nous penchons sur la scène didactique qui connait, de nos jours, une certaine montée des approches plurilingues, ou pédagogie intégrée, qui intègrent les langues étudiées de façon à

prendre appui sur le connu pour aborder le moins connu (De Pietro ; Balsiger, 2011 :

27).

Ces approches -qui croient en la possibilité de transferts positifs- renforcent les hypothèses didactiques formulées selon lesquelles la comparaison des langues pour en repérer les ressemblances et les différences améliorerait l'apprentissage. Que le transfert soit positif (transfert) ou négatif (interférence), le contact des langues est parfois générateur d'erreurs de divers types, que nous désignons par interférences. Des divers types, nous aborderons dans ce qui suit les interférences lexicales, objet de notre étude.

\section{Interférences lexicales : quelles particularités ?}

9 Les interférences interlinguales définies ci-dessus se manifestent dans les divers domaines de la langue, d'où ses divers types. Nous distinguons les interférences phonétiques, grammaticales, lexicales..., voire des interférences culturelles citées par Galisson et Coste (1976). Si les interférences phonétiques se rapportent à la transposition des phonèmes de la $\mathrm{L}$. source vers la $\mathrm{L}$. cible et que les interférences grammaticales concernent celle des règles, les interférences lexicales consistent en des unités lexicales de la L. source introduites -à tort- dans une production en L. cible (la langue étrangère apprise). Sur cette définition, de nombreux auteurs se montrent unanimes. Ainsi, Medane affirme que ce

transfert interlingual se fait lorsqu'un terme donné appartenant à la langue source fait intrusion dans la langue cible (2015). 
Dans le même sens, Galisson et Coste (1976) conçoivent les interférences comme un emploi de mots impropres dû au contact des langues. Ils associent le concept « interférence lexicale » à celui de « faux amis » qui désigne les mots qui existent dans deux langues différentes sans pour autant avoir la même signification; d'où les emplois erronés.

Jusqu'ici, le concept "interférence lexicale » dont il est question semble clairement défini. Il convient cependant de le distinguer des deux concepts « emprunt lexical » et « alternance codique » pour éviter toute confusion. En effet, l'interférence lexicale est un phénomène distinct de l'emprunt : le premier est individuel alors que le second est collectif. Loubier (2011) et Medane (2015) expliquent que le mot emprunté finit par s'imposer par l'usage, ce qui ne fausse pas son introduction dans les productions de la L. cible. Il n'en est pas de même pour l'interférence lexicale qui relève d'un emploi individuel généré par une compétence lexicale restreinte.

Outre sa distinction de l'emprunt, l'interférence lexicale est également distinguée de l'alternance codique. Par alternance de codes ou code-switching, nous désignons depuis les travaux de Gumperz (1989)- le fait d'alterner et de mêler les langues, dans des discours, en employant des expressions (mots, locutions ou phrases) d'une langue pour s'exprimer dans une autre. Cette définition du concept ne se prononce pas clairement sur la différence entre ces deux phénomènes inconscients et involontaires : interférence lexicale et alternance codique. Nous nous référons alors à Hassan (1974) qui oppose l'alternance lexicale, un phénomène résultant d'une incompétence linguistique, à l'alternance codique, un phénomène résultant d'une maîtrise équilibrée des langues apprises. Autrement dit, les interférences sont fréquentes dans les énoncés oraux ou écrits des apprenants alors que le code-switching se manifeste dans les énoncés des locuteurs ayant atteint un bilinguisme équilibré.

En somme, la perspective didactique fournit une définition assez claire de l'interférence interlinguale lexicale. Celle-ci renvoie en effet à un emploi individuel, volontaire ou inconscient, des mots de la langue source dans les productions orales ou écrites de la langue étrangère en cours d'apprentissage.

\section{Quelles en sont les raisons?}

12 Pour mieux comprendre les interférences interlinguales lexicales, nous ne limitons pas notre étude à la définition du concept. Nous aborderons les deux raisons essentielles qui les expliquent ; en nous inspirant la littérature scientifique en la matière.

\section{Compétence limitée}

13 Nous retrouvons cette première raison chez des auteurs tels que Hamers (1997) et Hagège $(1982,1996)$, qui imputent les interférences à une compétence limitée en langue étrangère. Dans ce sens, Hamers avance que

l'interférence se manifeste surtout chez des locuteurs qui ont une connaissance

limitée de la langue qu'ils utilisent » $(1997: 178)$.

Les locuteurs auxquels nous nous intéresserons sont des apprenants qui transfèrent les traits de la langue maternelle -ou d'une langue apprise antérieurement- vers la langue étrangère cible ; ce qui rejoint le transfert négatif évoqué par Öztokat (1993). 
Cependant, cette première raison -qui réduit les interférences à des signes d'incompétence linguistique- est actuellement dépassée. Ces erreurs -qui explicitent les stratégies mobilisées dans des situations-problèmes- relèvent en effet des interlangues des apprenants, qui sont constituées de leurs connaissances intermédiaires au cours de leur apprentissage d'une LE (Galligani, 2003).

\section{Psychotypologie}

14 Ringbom (1987) considère que la psychotypologie joue un rôle considérable dans les interférences lexicales. Selon Odlin, repris par Singleton et ó Laoire (2006) :

Le transfert proviendra très probablement du jugement que l'apprenant fait (consciemment ou inconsciemment) d'une similarité partielle ou totale entre des structures particulières appartenant à une langue précédemment acquise et des structures de la langue cible (Odlin, 1989 : 142).

15 Cette ressemblance typologique, établie également avec des langues apprises ultérieurement, est, selon Hatem, un des facteurs à l'origine des interférences interlinguistiques. Dans son article intitulé «L'interférence (inter)linguistique pour pallier le manque d'efficacité dans l'apprentissage des langues étrangères ", cet auteur traite la similitude typologique (au niveau de la forme) établie par les apprenants entre les deux langues étrangères apprises dans le contexte algérien : le français et l'anglais, qui les conduit à angliciser des mots français. Mentionnons que ces deux systèmes linguistiques, généalogiquement distants, sont lexicalement connectés étant donné que l'anglais « a emprunté la moitié de son vocabulaire au français » (Martinet, 1994, In Robert, $2008: 16)$.

Ces deux raisons -qui élucident certainement les origines des interférences lexicalessemblent étroitement liées étant donné que la perspective psychotypologique constitue également une stratégie investie pour résoudre une situation-problème: les apprenants établissent, à raison ou à tort, une ressemblance entre deux langues en cas de difficultés. Mentionnons que ces interférences lexicales pourraient être dues à d'autres facteurs tels que la contamination orthographique (Cuq, 2003) ci-dessus évoquée.

\section{A quelle langue les apprenants recourent-ils ?}

Les interférences lexicales constatées dans les productions des apprenants ne proviennent pas que de leur première langue, la langue maternelle. Toute langue constituant leur répertoire langagier pourrait provoquer des erreurs interlinguales.

Certains auteurs culpabilisent la langue maternelle (LM) : celle-ci présente à leurs yeux une source de fautes et de difficultés. Ainsi, Kannas (1994) estime que les interférences sont dues à la première langue apprise, c'est-à-dire la LM. De leur côté, Mackey (1976) et Öztokat (1993) -en définissant les interférences comme des transferts négatifs de la LM vers la langue cible- reconnaissent le rôle joué par la première langue dans l'apparition des erreurs dans la langue cible. Il en est de même pour Medane (2015) qui, dans son étude centrée sur des apprenants algériens, conclut que les erreurs repérées dans les discours produits en FLE sont dues à la première langue (arabe).

Contrairement à ces auteurs, Galisson et Coste (1976) estiment que la langue maternelle ne constitue pas la source unique d'erreurs étant donné qu'une langue étrangère 
antérieurement apprise pourrait provoquer des interférences dans les productions des apprenants. Dans son article contextualisé «Interférences lexicales entre deux langues étrangères : anglais et français ", şavlı (2009) précise que les étudiants turcs, qui ont la langue anglaise et la langue française comme langues étrangères, transfèrent en français les traits orthographiques de la langue anglaise.

Tout comme la langue précédemment apprise, une langue ultérieurement apprise pourrait influencer la L. cible. Nous illustrons cette affirmation par le travail de Hatem, ci-dessus cité, qui révèle la présence courante des mots anglicisés dans les productions des apprenants algériens en FLE.

Alors, les interférences lexicales ne résultent pas uniquement de la LM étant donné qu'une langue étrangère, qu'elle soit antérieurement ou ultérieurement apprise, pourrait laisser ses traces dans les écrits des apprenants en FLE.

\section{Méthodologie}

En vue de cerner la question des interférences lexicales chez les apprenants algériens du cycle moyen (4AM), nous avons procédé en premier lieu au repérage de ces erreurs dans les cinquante (50) productions collectées, qui constituent le corpus de notre étude. Suite à ce repérage, nous avons classé les erreurs interférentielles dans deux catégories : la première regroupe les unités lexicales de la langue arabe présentes dans les écrits (LM) alors que la seconde est constituée de celles de la langue anglaise (LE2). Les résultats obtenus seront présentés puis analysés dans les éléments qui suivent.

Nous précisons que les productions collectées, qui ne sont pas longues, répondent à deux consignes : description d'une fête traditionnelle et explication des avantages du téléphone mobile.

\section{Résultats}

Contrairement aux affirmations de Klinkenberg (1999), les interférences lexicales, ne sont pas nombreuses dans les productions de nos apprenants. Dans les cinquante (50) textes rédigés en FLE, seulement dix-huit (18) interférences lexicales sont repérées. Nous représentons dans le graphique ci-dessous la répartition de ces interférences sur les deux langues. 


\section{Interférences lexicales}

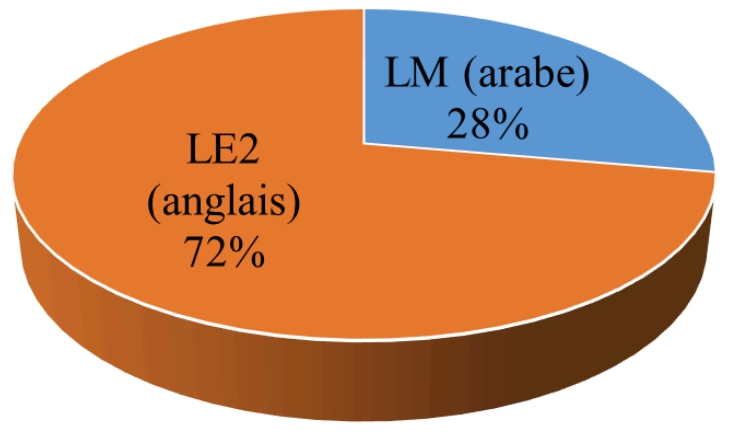

- LM (arabe) - LE2 (anglais)

Ce graphique qui représente les résultats de notre étude révèle que le nombre des mots anglais intégrés dans les productions des apprenants est plus élevé. Certains mots arabes, moins nombreux, sont également présents. De ce fait, les interférences lexicales sont dues aux deux langues présentes dans le milieu scolaire de ces apprenants : à la première langue (LM) et à la deuxième langue étrangère (LE2). Pourquoi nos apprenants recourent-ils à ces deux langues?

\section{Analyse et discussion}

Les mots anglais qui figurent dans les textes des apprenants sont à classer dans deux catégories. Les premières rassemblent les mots qui sont graphiquement et sémantiquement différents dans les deux langues comme in (au lieu de la préposition dans) et $a$ (à la place de un article indéfini). Quant à la deuxième, elle regroupe des mots lexicalement similaires à ceux de la langue française. Nous y incluons les mots : problem, no, dangerous, lesson, class, example et traditional. Contrairement aux unités lexicales de la première catégorie, ces unités-ci sont étymologiquement liées aux mots français problème, non, dangereux, leçon, classe, exemple et traditionnel. Dans cette situation, considérons-nous ces erreurs comme des interférences lexicales?

Si nous nous référons à la conception de Galisson et Coste (1976), évoquée supra, qui associent interférences lexicales aux faux amis, considérer ces erreurs comme interférences deviendra injustifié étant donné que la similitude orthographique des mots est renforcée par une similitude sémantique. Cependant, le recours des apprenants à ces mots demeure, à nos yeux, erroné en raison de la distance orthographique (leçon et lesson) et phonétique (dangereux et dangerous) entre les unités des deux langues.

Tenant compte de cette similitude, nous rejoignons donc la position des auteurs Ringbom, Odlin, Hatem... qui assignent les interférences lexicales dans les écrits en FLE à la psychotypologie. En effet, les apprenants établissent des similitudes graphiques entre les mots des deux systèmes linguistiques, qui les poussent à recourir à la LE2. En 
plus de la similitude graphique, notons la similitude phonétique entre certaines unités lexicales (problem/problème, class/classe, lesson/leçon...) qui pourrait générer une confusion orthographique ou une contamination (Cuq, 2003). Dans ces cas-ci, même des apprenants conscients des différences entre les deux systèmes linguistiques en question pourraient commettre ces erreurs interférentielles. Il est important que les erreurs de cette catégorie, qui semblent peu alarmantes, soient prises en charge par les enseignants pour éviter la fixation de formes incorrectes chez leurs apprenants, autrement dit la fossilisation.

Qu'elles soient conscientes ou inconscientes, ces transgressions nuisent à la correction linguistique des énoncés produits, sans en altérer le contenu sémantique et la fonction communicative. En s'appuyant sur leurs interlangues, élevées par Besse et Porquier (1991) au rang de compétences de communication, les apprenants ont élaboré des productions intelligibles en dépit des erreurs interférentielles constatées.

Si la similitude lexicale investie dans ce qui précède a permis la construction d'énoncés intelligibles, sa généralisation à des mots étymologiquement distants (un remplacé par $a$, dans remplacé par in) a abouti à des emplois incorrects, générateurs de confusions sémantiques.

La LE2 de nos apprenants ne constitue pas, cependant, la seule source d'interférences lexicales dans les productions des apprenants en FLE dans la mesure où ceux-ci se résignent parfois à un emploi volontaire des unités lexicales de leur LM, l'arabe. Conscients que cet emploi est incorrect vu la distance lexicale des deux systèmes linguistiques (arabe et français), ils choisissent des unités lexicales étrangères au FLE pour la communication de leurs idées.

23 Les interférences dues à la LM sont également réparties dans deux catégories. La première regroupe des mots arabes transcrits en français (tarikh pour Histoire) auxquels les apprenants recourent pour assurer la continuité sémantique de leurs énoncés. Quant à la seconde, elle comprend des mots du " français cassé » qui sont introduits en tant que mots français (roba au lieu de robe). Considérés comme des mots français, des mots comme roba sont en réalité des mots empruntés au français mais arabisés : ils ont subi des changements particuliers à la langue arabe (la forme féminine).

Contrairement aux interférences dues à une similitude lexicale, les unités lexicales arabes choisies par les apprenants révèlent une stratégie mobilisée pour résoudre une situation-problème : la langue maîtrisée (LM) devient une stratégie de communication en FLE.

Dues à la LE2 ou la LM, les interférences lexicales relevées dans les productions de nos apprenants sont peu nombreuses, ce qui contredit certaines affirmations. Le nombre restreint de ces intrusions dans les énoncés rédigés en FLE s'explique par des facteurs tels que le temps imparti aux tâches de rédaction qui permet aux apprenants de chercher des mots plus appropriés, notamment que les enseignants recommandent souvent l'emploi des unités lexicales propres au FLE. Nous ne pouvons pas nier, à ce niveau, certaines pratiques enseignantes qui sanctionnent les énoncés intégrant des mots arabes, ou des mots anglais, notamment ceux distants des mots français. Au lieu de sanctionner le recours, volontaire ou inconscient, à la LE2 et à la LM dans les productions pour éviter les interférences lexicales, les enseignants doivent observer ces erreurs et les comprendre pour sensibiliser les apprenants aux ressemblances et aux divergences entre les langues dont ces derniers disposent. Il s'agit donc de porter un regard positif sur la diversité des langues présentes dans le milieu scolaire, et sur les 
interférences lexicales qui pourraient être investies pour proposer des enseignements adaptés aux besoins authentiques des apprenants.

Pour conclure, nous précisons que les interférences lexicales, peu fréquentes dans les rédactions des apprenants algériens en FLE, sont dues à deux langues : LM et LE 2 . Conformément à nos hypothèses, la LM -une stratégie de compensation- ne constitue pas la seule source de ces erreurs interlinguales dans les productions analysées car les unités lexicales de la LE2 y sont plus courantes en raison de la similitude lexicale des deux systèmes linguistiques (français et anglais) et de la contamination (Ringbom, 1987 ; Cuq, 2003...).

\section{BIBLIOGRAPHIE}

Comprendre les origines de ces interférences chez nos apprenants est nécessaire pour que les enseignants agissent convenablement à travers des pratiques appropriées. Ces derniers doivent accorder une attention bienveillante aux intrusions linguistiques dans leurs classes de FLE, voire les investir pour des apprentissages plus significatifs. Par quelles pratiques les enseignants pourraient-ils alors remédier au problème des interférences interlinguales dans les écrits en FLE ?

Agues Martins, M-R., « Les phénomènes d'interférence linguistique dans l'enseignement/ apprentissage du français à Praia. Maîtrise en études françaises ", Maitrise en études françaises, ISE, 2007.

Besse, H., Porquier, R., Grammaire et didactique des langues, Langue et apprentissage des langues, Paris : Hatier/Didier, 1991.

Cuq, J-P., Dictionnaire de didactique du français langue étrangère et seconde, Paris : CLE International, 2003.

De Pietro, J-F., Balsiger, C., « Quand la diversité linguistique devient la norme », Educateur : Approches plurilingues, N13, 2011, 26-29.

Galisson, R., Coste, D. (Dirs)., Dictionnaire de didactiques des langues, Paris : Hachette, 1976.

Galligani, S., « Réflexion autour du concept d'interlangue pour décrire des variétés non natives avancées en français ", Linx, N 49, 2003, 141-152.

Gumperz, J.J., Sociolinguistique Interactionnelle : une approche interprétative, France :

Université de la Réunion/Le Harmattan, 1989.

Hagège, C., L'enfant à deux langues, Paris : Editions Odile Jacob, 1996.

Hagège, C., La Structure des langues, Paris : Presse Universitaire de France, 1982.

Hamers, J.-F., «Interférence », Dans Moreau M.-L. (Ed.), Sociolinguistique : concepts de base, Bruxelles : MARDAGA, 1997.

Hamers, J.-F., Blanc M., Bilingualité et bilinguisme, Bruxelles : Pierre MARDAGA, 1983. 
Hassan, A., « Interférence, linguistique contrastive et sa relation avec l'analyse des fautes », La pédagogie des langues vivantes, N 5, 1974, 168-173.

Hatem Y., «L'interférence (inter)linguistique pour pallier le manque d'efficacité dans l'apprentissage des langues étrangères ", Disponible sur [www.academia.edu], Consulté le 07/03/2019.

Kannas, C., Dictionnaire de linguistique et des sciences du langage, Paris : Larousse, 1994.

Klinkenberg, J-M., Des langues romanes, Bruxelles : Ducolot, 1999.

Loubier, C., De l'usage de l'emprunt linguistique, Québec : Office québécoise de la langue française, 2011, ISBN Version électronique : 978-2-550-61626-9.

Mackey, W. F., Bilinguisme et contact des langues, Paris : Edition Klincksieck, 1976.

Martinet, A. Préface à L'aventure des langues en Occident, de H. Walter, Laffont, 1994.

Medane, H., «L'interférence comme particularité du « français cassé » en Algérie », TIPA

(Travaux Interdisciplinaires sur la Parole et le Langage) [En ligne], 2015, Disponible sur [https:// journals.openedition.org/tipa/1394], DOI : 10.4000/tipa.1394, Consulté le 18/09/2019.

Odlin, T., Language Transfer : Cross-linguistic Influence in Language Learning, Cambridge : Cambridge University Press, 1989.

Öztokat, N., « Analyse des erreurs/ analyse contrastive in Grammaire et Didactique des langues ", Eskişehir, Anadolu Üniversitesi yayın, N 590, 1993, 66-76.

Ringbom, H., The Role of the First Language in Foreign Language Learning, Multilingual Matters, 1987.

Robert, J-M., «L'anglais comme langue proche du français ? », Éla. Études de linguistique appliquée, N 149, 2008, 9-20.

Robert, J-P., Dictionnaire pratique de didactique du FLE, Paris : OPHRYS, 2002.

şavli, F., « Interférences lexicales entre deux langues étrangères : anglais et français », Synergies Turquie, N 2, 2009, 179-184.

Singleton, D., Ó Laoire, M., « Psychotypologie et facteur L2 dans l'influence translexicale : une analyse de l'influence de l'anglais et de l'irlandais sur le français L3 de l'apprenant », Acquisition et Interaction en Langue Etrangère, N 24, [en ligne], 2006, 101-117.

Weinrich, U., Languages in contact, findings and problems, New York : Linguistic Circle of New York, 1953.

\section{RÉSUMÉS}

Cette étude porte sur les origines des interférences lexicales chez les apprenants de FLE, dont le répertoire est constitué de : l'arabe (LM), le français (LE1) et l'anglais (LE2). L'analyse de leurs écrits a révélé que la LE2, ultérieurement apprise, est la première source d'interférences vu sa similitude lexicale avec le FLE. La LM, peu fréquente, est investie en tant que stratégie de compensation.

This study concerns the origins of lexical interferences in learners of French as a foreign language,_whose repertoire consists of : Arabic (NL), French (FL 1) and English (FL 2). Analysis of their writings reveals that the FL2, language they learned later, is the first source of interference 
given its lexical similarity with the French language. The NL, infrequent, is used as a compensation strategy.

INDEX

Keywords : Lexical interference, written production, French FL, NL, FL 2

Mots-clés : Interférence lexicale, production écrite, FLE, LM, LE 2

\section{AUTEUR}

\section{ASSIA LAIDOUDI}

MAB, Université de MSILA, Laboratoire de Recherche SELNoM, Algérie. 